\title{
Option
}

\section{Protection intégrée des cultures maraîchères sous serre : expérience et atouts pour un contexte en évolution *}

\author{
Philippe Claude Nicot \\ Institut national de la recherche agronomique \\ (Inra), \\ Centre d'Avignon, \\ Unité de pathologie végétale, \\ Domaine Saint Maurice, \\ BP 94 \\ 84143 Montfavet cedex \\ <philippe.nicot@avignon.inra.fr>
}

\begin{abstract}
Résumé
La serre constitue un environnement particulièrement favorable au développement de divers ennemis des cultures. C'est aussi dans ce système de production que des approches phytosanitaires combinant différents types d'interventions sont utilisées depuis de nombreuses années, notamment pour la lutte contre certains ravageurs. La dénomination de ces approches (initialement " lutte intégrée ") a suivi l'évolution conceptuelle de la gestion phytosanitaire des systèmes agricoles, et les termes "protection (biologique et) intégrée ", " production intégrée ", "Agriculture raisonnée " sont de plus en plus associés aux cultures maraîchères sous abri. Cependant, le contexte économique tendu des filières et la diversité et complexité technique de la production sous serre ont-ils permis une évolution équivalente au niveau des pratiques? Alors que la mise en œuvre de la lutte chimique, même raisonnée, rencontre des difficultés croissantes, la palette d'outils alternatifs mobilisables pour la gestion de certains ravageurs et agents pathogènes sous abri s'est progressivement étoffée. Le déploiement coordonné de cet arsenal dans le cadre d'une protection intégrée (tenant compte de l'ensemble des contraintes phytosanitaires) reste cependant largement empirique et manque encore de robustesse face à certaines perturbations du système (émergence de nouveaux bioagresseurs, évolution des contraintes réglementaires ou socio-économiques). Par ailleurs, comme pour la lutte chimique ou l'utilisation de résistance variétale, certaines études suggèrent que la durabilité d'autres méthodes de lutte (biologique, physique) pourrait aussi être remise en question par des bioagresseurs à fort potentiel de variabilité. Dans une perspective de développement durable, pour lequel la production sous serre présente des atouts indéniables, il paraît souhaitable d'envisager directement une démarche de recherche axée sur la production intégrée, qui prenne en compte la globalité des contraintes (agronomiques, mais aussi socio-économiques) de la filière. Ce type d'approche multidisciplinaire représente un défi que des équipes Inra (Institut national de la recherche agronomique) et universitaires ont choisi de relever, en association avec divers organismes interprofessionnels dans le cadre du programme fédérateur "Agriculture et développement durable ".
\end{abstract}

Mots clés : culture maraîchère ; culture sous serre ; lutte intégrée ; production intégrée ; protection intégrée.

Thèmes : productions végétales ; méthodes et outils.

\section{Abstract \\ Integrated protection of greenhouse vegetable crops: benefits from experience and valuable assets within a fast-changing context}

Greenhouses have a micro-climate that is more humid and warmer than the outside environment. These conditions are particularly conducive to development of a variety of plant pests. In this agrosystem, plant health management has relied for many years on combinations of diverse control methods directed at various pests. This approach (initially 
referred to as "integrated control") has followed the general conceptual evolution of other agrosystems, and the terms "integrated (and biological) protection", "integrated production", and in France "Agriculture raisonnée", have been increasingly associated with greenhouse vegetable production. However, is it possible for similar development to take place at a practical level, considering the increasingly competitive economic situation and an increasingly complex production scheme? While chemical control has become increasingly difficult, the array of alternative control methods against various pests and pathogens of greenhouse crops has progressively broadened. However, the coordinated deployment of this arsenal in the context of integrated protection (taking into account all plant health constraints in the greenhouse) remains largely empirical and still lacks robustness in confronting a variety of disturbances within the system (emergence of new pests, evolution of legal or economic and social constraints). Furthermore, several studies on durability suggest that in the same way chemical control and the deployment of resistant varieties suffers diminishing effect with recurrent use, other control methods (biological, physical) could also lose their effectiveness against particularly variable plant pests or pathogens. In a perspective of durable development, for which greenhouse production has specific advantages, research should be focused on Integrated Production to take as many of the constraints into account in the production system as possible. Such a multidisciplinary approach represents a challenge that a consortium of Inra (Institut national de la recherché agronomique) and University research teams, together with groups representing growers' associations, has taken up within the framework of the French national program "Agriculture and Sustainable Development".

Key words: integrated control; greenhouse crops; integrated production; integrated protection; vegetable growing.

Subjects: vegetal productions; tools and methods.

a culture sous abri fournit aujourd'hui une part essentielle du marché de frais pour les légumes-fruits tels que la tomate. (Jeannequin et al., 2005). Ce système de production est extrêmement intensif, en particulier en ce qui concerne les cultures hors sol en serre chauffée. A-t-il sa place dans un contexte de développement durable, auquel on associe généralement un mode de culture plutôt extensif? C'est la question à laquelle tente de répondre un projet multidisciplinaire porté par l'Institut national de la recherche agronomique (Inra) dans le cadre du programme fédérateur national "Agriculture et développement durable ". La culture sous abri présente certes des faiblesses, mais aussi des atouts significatifs. Un de ses atouts majeurs est la place importante et le succès de la mise en œuvre de protection intégrée pour gérer la santé des cultures. Cette communication se propose d'examiner la situation de la protection intégrée sous serre et de dégager quelques perspectives sur son évolution possible.

\section{Évolution conceptuelle de la protection intégrée sous serre}

Le concept d'une gestion raisonnée et intégrée des problèmes phytosanitaires a été fortement porté et popularisé par les actions de l'Organisation internationale de la lutte biologique (OILB). Depuis la création (dès 1968) d'un groupe de travail "Lutte intégrée sous serre" au sein de cette organisation (Boller et al., 2006), l'évolution conceptuelle de la gestion phytosanitaire sous serre a suivi de près celle d'autres systèmes de cultures, avec la prise en compte successive de trois niveaux d'intégration : i) le pathosystème individuel (lutte intégrée contre un bioagresseur) ; ii) un pathosystème multiple (protection intégrée contre les principaux bioagresseurs de la culture); et iii) l'ensemble du système de culture (production intégrée). Comme en témoi- gnent des documents de l'OILB (Steiner, 1977 ; Boller et al., 2006), la réflexion conceptuelle sur ce dernier niveau d'intégration (incluant même des références à une "agriculture viable ") a démarré il y a au moins une trentaine d'années au sein de la communauté des chercheurs du domaine phytosanitaire.

Cependant, une analyse des publications récentes de cette communauté suggère que les travaux de recherche sont encore très majoritairement axés sur le premier niveau d'intégration. Par exemple, 60 à $80 \%$ des communications aux derniers colloques organisés par l'OILB sur la protection intégrée des cultures sous abri (Castañé et Hanafi, 2003; Enkegaard, 2005) et la gestion intégrée des maladies et des ravageurs dans les agrosystèmes (Elad et al., 2004) décrivaient des études sur un pathosystème unique. Par contraste, 17 à $34 \%$ des communications concernaient la gestion simultanée de plusieurs bioagresseurs, et une proportion très faible (0-10\%) était axée sur des interactions entre la composante phytosa- 
nitaire et au moins un aspect des autres éléments de l'agrosystème. Cette proportion de travaux concernant une intégration de niveau 3 est plus élevée si l'on pratique la même analyse pour une revue focalisée sur des thématiques agronomiques $(12,7$ et $24,7 \%$, respectivement, pour Agronomie, en 2004 et Agronomy and Sustainable Development, en 2005). Cependant, elle reste très modeste $(<5 \%)$ si l'on exclut les publications sur le devenir de produits phytosanitaires dans l'environnement.

Parmi les études de phytoprotection relevant du niveau d'intégration 1 dans l'exemple considéré (colloques OILB), quelques-unes sont focalisées sur les interactions entre un bioagresseur, sa plante hôte et l'environnement (épidémiologie et dynamique des populations de bioagresseurs, modélisation). Mais les facteurs d'efficacité de méthodes de lutte (résistance variétale, lutte chimique, lutte biologique, méthodes physiques et mesures culturales de prophylaxie) et leur interaction avec les diverses composantes du pathosystème constituent l'objet d'étude dominant. Les interactions possibles (compatibilité, synergie) entre différents types d'interventions phytosanitaires pour la lutte intégrée contre un bioagresseur donné sont également assez bien représentées.

\section{Situation opérationnelle : l'exemple de la tomate}

En termes opérationnels, on considère généralement que les cultures sous abri, en particulier de concombre et de tomate, sont majoritairement gérées en protection intégrée. Diverses sources s'accordent sur une estimation d'environ 150 hectares de concombre et 1000 hectares de tomate (par exemple, www.tomates-de-france. com), représentant respectivement 80 et $50 \%$ des exploitations françaises. Pour la tomate, la proportion approcherait les $80 \%$ en considérant uniquement les serres lourdes chauffées.

Cependant, il n'est pas certain que ces chiffres donnent une image fidèle de la situation sur le terrain. En effet, comment distinguer parmi les exploitations recensées, celles qui mettent en œuvre une gestion intégrée globale de la santé de leur culture, et celles qui font usage d'auxiliaires pour lutter contre un des ravageurs pendant une partie de la saison de culture? De fait, la mise en ouvre de la protection intégrée est très technique et la longue durée de la saison de culture, qui peut atteindre 11 mois, est propice à des dérapages et au recours à des traitements chimiques correctifs qui compromettent ensuite l'ensemble de la protection intégrée.

Par ailleurs, la lutte chimique reste un moyen opérationnel incontournable contre certains ravageurs importants (acariens, thrips, par exemple - tableau 1) et contre certaines maladies (tableau 2). Il faut noter que si des résistances variétales individuelles existent contre la plupart des maladies importantes sous serre, il n'existe aucune variété résistante ou tolérante à l'ensemble d'entre elles. Actuellement, aucun produit de lutte biologique n'est homologué en France contre ces maladies, mais les choses pourraient changer dans un proche avenir car des produits sont déjà utilisés à l'étranger.

Une part importante de la complexité de la protection intégrée est la gestion des interactions possibles entre bioagresseurs (notamment, par exemple, la transmission de virus par les insectes) et surtout entre les méthodes de lutte qui sont mises en œuvre pour les combattre. C'est un champ de recherche très actif dont les résultats les plus finalisés sont rapidement exploités par la filière. C'est le cas par exemple de l'établissement de listes de compatibilité de produits phytosanitaires avec divers agents de lutte biologique. Si l'on s'intéresse maintenant au niveau d'intégration englobant la totalité de l'agrosystème, qui constitue le domaine d'action au quotidien pour le serriste, deux points doivent être considérés : i) le premier est la très grande complexité des décisions qui doivent être prises par le serriste, souvent au jour le jour, avec parfois des situations ou des objectifs aboutissant à des consignes contradictoires à gérer; ii) le second est la place réduite de la gestion phytosanitaire au sein de cet ensemble, tant en termes de volume de décisions à prendre qu'en termes de proportion du coût de production.

Face à cette complexité, différents systèmes d'aide à la décision ont été développés. Ils sont généralement axés sur la gestion optimisée de certains facteurs de production tels que le climat ou la fertirrigation. C'est le cas par exemple du programme "Serriste", développé par l'Inra, ou d'un logiciel beaucoup plus simple développé à l'Obio Agricultural Research and Development Center (OARDC) et disponible en ligne (www.oardc.ohiostate.edu/ hydroponics/DecisionAnalysis/decision

\section{Tableau 1. Principaux ravageurs de la tomate sous serre et méthodes de lutte pratiquées.}

Table 1. Main pests of greenhouse tomato and control methods.

\begin{tabular}{|c|c|}
\hline Principaux ravageurs & Méthodes de lutte \\
\hline $\begin{array}{l}\text { Aleurodes } \\
\text { (Bemisia tabaci, Trialeurodes } \\
\text { vaporariorum) }\end{array}$ & $\begin{array}{l}\text { Parasitoïdes (Encarsia, Eretmocerus) ; prédateurs } \\
\text { (Macrolophus); entomopathogènes (Lecanicillium, } \\
\text { Paecilomyces); insecticides }\end{array}$ \\
\hline $\begin{array}{l}\text { Acarien } \\
\text { (Tetranychus urticae) }\end{array}$ & Acaricides \\
\hline $\begin{array}{l}\text { Mineuses } \\
\text { (Liriomyza bryoniae, L. trifolii, } \\
\text { L. huidobrensis) }\end{array}$ & $\begin{array}{l}\text { Parasitoïdes (Dacnusa, Diglyphus) et contrôle } \\
\text { naturel ; insecticides }\end{array}$ \\
\hline $\begin{array}{l}\text { Pucerons } \\
\text { (ex: Myzus persicae, } \\
\text { Aphis gossypii, } \\
\text { Macrosiphum euphorbiae) }\end{array}$ & $\begin{array}{l}\text { Parasitoïde (Aphelinus) ; prédateurs (Aphidoletes) et } \\
\text { contrôle naturel ; insecticides }\end{array}$ \\
\hline $\begin{array}{l}\text { Lepidoptera } \\
\text { (ex: Chrysodeixis chalcites, } \\
\text { Lacanobia oleracea, } \\
\text { Spodoptera littoralis) }\end{array}$ & Pathogène (Bacillus thuringiensis); insecticides \\
\hline $\begin{array}{l}\text { Thrips } \\
\text { (Frankliniella occidentalis, } \\
\text { Thrips tabacl) }\end{array}$ & Entomopathogène (Lecanicillium) ; insecticides \\
\hline $\begin{array}{l}\text { Nématodes } \\
\text { (ex: Meloidogyne spp.) }\end{array}$ & Variétés résistantes ou tolérantes ; culture hors-sol \\
\hline
\end{tabular}


Tableau 2. Principales maladies de la tomate sous serre et méthodes de lutte pratiquées.

Table 2. Main diseases of greenhouse tomato and control methods.

\begin{tabular}{|c|c|}
\hline Principales maladies & Méthodes de lutte ou de prévention \\
\hline $\begin{array}{l}\text { Pourriture grise } \\
\text { (Botrytis cinerea) }\end{array}$ & $\begin{array}{l}\text { Gestion climatique ; méthodes culturales ; fongicides ; } \\
\text { lutte biologique? }\end{array}$ \\
\hline $\begin{array}{l}\text { Oïdium } \\
\text { (Oidium neolycopersici, } \\
\text { Leveillula taurica) }\end{array}$ & $\begin{array}{l}\text { Fongicides (+ "lampe à soufre ») ; variétés } \\
\text { résistantes ; lutte biologique? }\end{array}$ \\
\hline $\begin{array}{l}\text { Fusariose } \\
\text { (Fusarium oxysporum } \\
\text { lycopersici) }\end{array}$ & $\begin{array}{l}\text { Variétés résistantes ; culture hors sol ; fongicides ; lutte } \\
\text { biologique? }\end{array}$ \\
\hline $\begin{array}{l}\text { FORL } \\
\text { (Fusarium oxysporum } \\
\text { radicis-lycopersici) }\end{array}$ & $\begin{array}{l}\text { Variétés résistantes ; culture hors sol ; hygiène ; } \\
\text { fongicides ; lutte biologique? }\end{array}$ \\
\hline $\begin{array}{l}\text { Verticilliose } \\
\text { (Verticillium dahliae) }\end{array}$ & Variétés résistantes; culture hors sol \\
\hline $\begin{array}{l}\text { Cladosporiose } \\
\text { (Fulvia fulva) }\end{array}$ & Variétés résistantes; gestion climatique; fongicides \\
\hline Viroses & $\begin{array}{l}\text { Variétés résistantes/tolérantes (TMV, TSWV, TYLCV) ; } \\
\text { hygiène ; gestions des adventices ; gestion des } \\
\text { vecteurs }\end{array}$ \\
\hline
\end{tabular}

TMV : Tobacco mosaic virus, TSWV : Tomato spotted wilt tospovirus ; TYLCV : Tomato yellow leaf curl virus.

analysis.ht). Ce type de système est parfois directement intégré dans les logiciels commercialisés pour la gestion climatique des serres. Cependant, le couplage de modèles de production avec des modèles de protection phytosanitaire reste une tâche beaucoup plus ardue. Un exemple de programme prenant en compte les aspects phytosanitaires est celui développé par la Station expérimentale d'Agriculture Canada à Harrow dans l'Ontario (Clarke et al., 1999). Conçu sur la base d'une approche intégrée holistique, il comprend: un module de diagnostic des problèmes phytosanitaires (système expert), un module de contrôle (interventions phytosanitaires), une base de données (enregistrement et analyse des itinéraires techniques détaillés) et une boîte à outils (concentration de fertirrigation, propriétés psychrométriques de l'air). La dernière mise à jour est actuellement utilisée par 54 licenciés dans 16 pays (L. Shipp, comm. pers.).

\section{Durabilité}

\section{de la protection intégrée sous serre}

La durabilité de la protection intégrée dépend de la durabilité de l'efficacité des différentes composantes sur lesquelles elle s'appuie. Pour les cultures sous serre comme pour les autres systèmes de production, on peut trouver de nombreux exemples de résistance de certains bioagresseurs (ravageurs ou agents pathogènes) à divers pesticides, ou des exemples de contournement de résistance variétale. Dans ce dernier cas, les enjeux et difficultés de la sélection de résistance durable sont compliqués par la prise en compte souhaitable de bioagresseurs multiples et par la durée de vie limitée des variétés par rapport à la durée du travail de sélection. Une question de plus en plus souvent posée concerne la durabilité des autres méthodes de lutte (biologique ou culturales). Avec quelques exceptions telles que la résistance de lépidoptères à Bacillus thuringiensis ou plus récemment au virus de la granulose (Sauphanor et al., 2006) dans d'autres systèmes de culture, peu d'information est disponible actuellement.

Un autre aspect de la durabilité de la protection intégrée est sa robustesse face à des perturbations extérieures au système. Ces perturbations peuvent résulter de l'émergence de nouveaux problèmes phytosanitaires. Quelques exemples récents pour la tomate sous serre ont été observés, dans les années 1990 : Oidium neolycopercisi, le thrips californien et le virus qu'il transmet (Tomato spotted wilt tospovirus, TSWV), et plus récemment, le virus du Pepino, ainsi que Bemisia tabaci et les virus qu'elle transmet (Tomato yellow leaf curl virus TYLCV; Tomato Infectious Chlorosis Virus, TIC; Tomato Chlorosis Virus, ToCV, etc.). D'autres types de perturbations sont d'ordre réglementaire, impliquant notamment des produits phytosanitaires (directive européenne 91/414/CE, interdiction du bromure de méthyle) ou des pratiques culturales (obligation de recyclage des solutions nutritives en culture hors sol), ou bien résultent d'évolutions technologiques majeures du système de production (utilisation des bourdons pour la pollinisation). Toutes ont des conséquences sur la protection intégrée. Un des épisodes les mieux documentés pour les cultures sous abri est probablement la régression sensible des surfaces bénéficiant de lutte intégrée contre les ravageurs, entre 1990 et 1995, qui a fait suite à l'arrivée du thrips californien (Maisonneuve et al., 2003). Globalement, la culture de tomate a été relativement peu affectée, peut-être en partie parce que la palette de méthodes de lutte était suffisante pour maitriser cette perturbation, peut-être aussi en relation avec l'utilisation des bourdons pour la pollinisation, qui est peu compatible avec un retour vers le "tout chimique ". La culture de concombre a été la plus affectée, et la réversion très forte de la tendance à partir de 1997 a suivi le développement de méthodes de lutte intégrée contre ce problème phytosanitaire, dans lesquelles figurent de nouveaux agents de lutte biologique contre le thrips sur cette culture.

\section{Conclusions - Perspectives}

Dans un contexte de développement durable, la protection intégrée constitue un atout très important pour les cultures sous abri, et son succès est fréquemment mis en avant dans les démarches de communication de la filière (voir par exemple www.tomates-de-france.com). Comme les autres productions agricoles, les cultures sous abri ont beaucoup à gagner dans la démarche de prise en compte de l'ensemble du système de production. Le développement d'initiatives diverses de la part de groupements de producteurs a stimulé une réflexion sur la définition d'une agriculture raisonnée et le principe 
de la labellisation des exploitations (Paillotin, 2000) et sur le choix des référentiels (Beigbeder et Meynard, 2001). L'ensemble de ces travaux a été suivi de la formalisation réglementaire d'un "référentiel de l'agriculture raisonnée " en 2002 (Journal Officiel du 4 mai 2002) et d'une modification en 2005 (Journal Officiel du 20 avril 2005). Au sein des 98 exigences nationales figurant dans ce document, 16 concernent divers aspects de la protection des cultures, dont quatre seulement sont directement liés aux procédés de lutte contre les bioagresseurs. Ces points reprennent globalement les avancées de la protection intégrée, notamment sur le déclenchement des traitements, mais sans toutefois citer d'exigences spécifiques sur les méthodes de lutte à mettre en œuvre. En cela, ce référentiel apparaît moins exigeant que celui des cahiers des charges imposés par certains metteurs en marché ou distributeurs, et auxquels de nombreux serristes se soumettent pour la commercialisation de tomates et d'autres légumes frais. Et cependant, il semble que peu de serristes aient entrepris la démarche de qualification "Agriculture raisonnée ", comme en attestent les très faibles nombres d'exploitations référencées en productions légumières pour les principaux départements producteurs de tomates et autres légumes sous serres (http://www.farre.org/). Par ailleurs, le niveau d'exigence du référentiel de l'agriculture raisonnée est également très en deçà de celui de la production intégrée publié par l'OILB (El Titi et al., 1993 ; Boller et al., 2004). Dans une perspective d'exigences plus grandes, des progrès techniques sont encore nécessaires.

Parmi les besoins de recherche en protection intégrée, il parait nécessaire : i) de continuer à enrichir la palette d'outils tactiques, notamment en ce qui concerne une résistance variétale durable (si possi- ble contre de multiples bioagresseurs) et la lutte biologique ; le besoin de développement de produits commerciaux est particulièrement fort pour la lutte biologique contre les maladies; ii) de prendre en compte de façon précoce la dimension "durabilité " dans le développement de méthodes de lutte. Il s'agit de recherche fondamentale permettant de comprendre pour prévoir la durabilité d'une méthode de lutte; iii) de renforcer les efforts de modélisation de l'ensemble du fonctionnement d'un agrosystème, en vue de développer des systèmes d'aide à la décision et de permettre de tester des hypothèses tactiques. Cette approche nécessite une implication des disciplines socioéconomiques et de l'ensemble des acteurs de la filière. Elle devrait contribuer à la mise en œuvre d'une production sous abri encore plus respectueuse de l'environnement.

\section{Remerciements}

Merci à Gilles Ridray, Benoît Jeannequin (Inra, Alénya), Jean-Michel Leyre, Yannie Trottin-Caudal (Centre technique interprofessionnel des fruits et légumes, CTIFL, Balandran) et à Isabelle Mazal, Richard Brand (Groupe d'étude et de contrôle des variétés et des semences, Geves, Avignon) pour leur assistance dans la mise à jour des informations présentées dans les tableaux 1 et 2 .

\section{Références}

Beigbeder N., Meynard J.M. Aide à la définition d'un référentiel national de l'agriculture raisonnée et étude comparée des politiques et des pratiques des états membres de I'Union européenne relatives à l'agriculture raisonnée. 2001. http ://www.farre.org/QUALIFIC/ Reglementation/FONDEMEN.HTM.
Boller EF, van Lenteren JC, Delucchi V. International Organization for Biological Control of Noxious Animals and Plants: History of the first 50 years. Zürich : International Organisation for Biological Control of Noxious Animals and Plants (IOBC), 2006.

Boller EF, Avilla J, Joerg E, Malavolta C, Wijnands FG, Esbjerg P. Integrated production: principles and technical guidelines. 3rd ed. IOBCNWPRS Bull 2004 ; 27(2) : 1-49.

Castañé C, Hanafi A. Integrated control in protected crops, Mediterranean climate. IOBC/ WPRS Bull 2003 ; 26 (10) : 1-192.

Clarke ND, Shipp JL, Papadopoulos AP, et al. Development of the Harrow Greenhouse Manager: a decision-support system for greenhouse cucumber and tomato. Comput Electron Agric $1999 ; 24$ : 195-204.

EI Titi A, Boller EF, Gendrier JP. Integrated production: principles and technical guidelines. IOBCMPRS Bull 1993 ; 16 (1) : 1-96.

Elad Y, Pertot I, Enkegaard A. Management of plant diseases and arthropod pests by BCAs and their integration in agricultural systems. IOBCNWPRS Bull $2004 ; 27$ (8) : 1-429.

Enkegaard A. Integrated control in protected crops, temperate climate. IOBCWWRS Bull $2005 ; 28(1): 1-328$

Jeannequin B, Dosba F, Amiot-Carlin MJ. Fruits et Légumes. Caractéristiques et principaux enjeux. Collection " Un point sur les filières ". Paris : Inra éditions, 2005.

Maisonneuve JC, Capy A, Pelletier B, TrottinCaudal Y. Une culture pionnière en Protection Biologique et Intégrée: la tomate ; situation des superficies et évolution. In: Tomate sous abri. Protection intégrée, Agriculture Biologique. Paris: Centre technique interprofessionnel des fruits et légumes (CTIFL), 2003.

Paillotin G. L'agriculture raisonnée. Rapport au Ministre de l'Agriculture et de la Pêche. 2000. http ://www.farre.org/QUALIFIC/ Reglementation/FONDEMEN.HTM

Sauphanor B, Berling M, Toubon JF, Reyes M, Delnatte J, Allemoz P. Carpocapse des pommes : cas de résistance aux virus de la granulose. Phytoma - La Défense des Végétaux $2006 ;(590): 24-7$.

Steiner $\mathrm{H}$. Vers la production agricole intégrée. IOBCNPRS Bull $1977 ; 4: 1-153$. 